\title{
BMJ
}

\section{Mental capacity to make decisions on treatment in people admitted to psychiatric hospitals: cross sectional study}

\author{
Gareth S Owen, clinical researcher, ${ }^{1}$ Genevra Richardson, professor of law, ${ }^{2}$ Anthony S David, professor of \\ cognitive neuropsychiatry, ${ }^{1}$ George Szmukler, professor of psychiatry and society, ${ }^{3}$ Peter Hayward, \\ consultant clinical psychologist, ${ }^{4}$ Matthew Hotopf, professor of general hospital psychiatry ${ }^{1}$
}

${ }^{1}$ Department of Psychological Medicine and Psychiatry, Institute of Psychiatry, King's College London, London SE5 8AF

${ }^{2}$ School of Law, King's College London

${ }^{3}$ Department of Health Services and Population Research, Institute of Psychiatry, King's College London

${ }^{4}$ Maudsley Psychology Centre, Maudsley Hospital, London

Correspondence to: $\mathrm{M}$ Hotopf m.hotopf@iop.kcl.ac.uk

doi:10.1136/bmj.39580.546597.BE

\section{ABSTRACT}

Objective To estimate the prevalence of mental capacity to make decisions on treatment in people from different diagnostic and legal groups admitted to psychiatric hospital.

Design Cross sectional study.

Setting General adult acute psychiatric inpatient units. Participants 350 consecutive people admitted to psychiatric wards from the community over 16 months. Main outcome measure Mental capacity assessed by clinical interview and the MacArthur competence assessment tool for treatment.

Results Estimates of mental capacity were obtained on $97 \%(n=338)$ of the 350 people admitted. Of those an estimated $60 \%$ (95\% confidence interval $55 \%$ to $65 \%$ ) lacked mental capacity to make decisions on treatment. This proportion varied according to diagnosis, ranging from $97 \%(n=36)$ in people with mania to $4 \%(n=24)$ in people with personality disorder. Mental incapacity was common in patients admitted informally to the psychiatric wards ( $n=188 ; 39 \%, 32 \%$ to $46 \%$ ). Incapacity and detention are closely associated under non-capacity based mental health law.

Conclusions Mental incapacity to make decisions on treatment is common in people admitted to psychiatric wards from the community but cannot be presumed. It is usual in those detained under the Mental Health Act and common in those admitted voluntarily.

\section{INTRODUCTION}

The concept of mental capacity is central to modern medical law and applies to people with mental

Consecutive admissions from community $(n=350)$

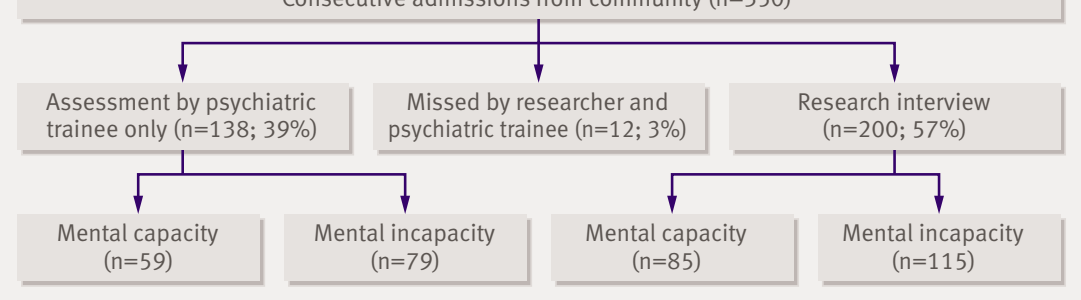

Flow of patients through study disorder. Its significance is linked to the principle of autonomy, which demands that an individual's autonomous decisions relating to the acceptance and refusal of medical treatment be respected. ${ }^{12}$ Provision for those who lack mental capacity exists in many developed legal systems. However, such provision typically stands alongside separate legal provisions, not capacity based, for the involuntary treatment of mental disorder. This dual legislative approach to people with mental disorder has raised ethical concerns about respect for the principle of autonomy and practical concerns about which legislative framework such people come under. ${ }^{3}$ Relevant, good quality studies in this area are limited. ${ }^{45}$ We describe the frequency of capacity to decide on key treatment decisions in adults consecutively admitted to a psychiatric hospital, and in diagnostic and legal (informal compared with involuntarily detained) subgroups.

\section{METHODS}

We included people consecutively admitted to three general adult psychiatric wards, serving a deprived inner city area, at the Maudsley Hospital, London between February 2006 and June 2007. Admitted people were identified by regular examination of electronic medical records and consultations with ward nursing staff. We excluded those from other catchment areas or transferred from other inpatient facilities.

Patients were approached for a research interview. Those assenting were given full details of the study. Written consent was obtained. We stopped the interview if there was any change in choice, or resistance. Patients were offered $£ 5$ ( $€ 6.3 ; \$ 9.8)$ for their time. Trained senior house officers who cared for the patients assessed the mental capacity of all people admitted. Non-consenting people or those who did not speak English were assessed only by psychiatric trainees. Assessments of mental capacity were done as close to admission as possible.

Assessment of mental capacity

From the medical records we obtained information on the patient's presenting problems, diagnosis 
Table 1|Personal, clinical, and legal characteristics of people admitted to psychiatric wards and assessed for mental capacity to make decisions on treatment. Values are numbers (percentages) unless stated otherwise

Variable

Mean (SD) age (years)

Sample $(n=350)$

Men $38(11.4)$

Ethnicity

\begin{tabular}{lc}
\hline White European & $157(44)$ \\
\hline Black African & $80(23)$ \\
\hline Black Caribbean & $62(18)$ \\
\hline Black other & $19(5)$ \\
\hline Other ethnic minority group & $32(9)$ \\
\hline Accommodation: &
\end{tabular}

Accommodation:

Living with partner or family 120 (34)

Living alone independently 143 (41)

Supported accommodation $53(15)$

No fixed abode $23(7)$

Unknown 11 (3)

Mean (SD) education (years) since age $10 \quad 7.0(3.0)$

Occupation:

$\begin{array}{ll}\text { Employed } & 60(17)\end{array}$

Student 10 (3)

Not economically active 274 (78)

Unknown 6(2)

Marital status:

Single $291(83)$

Married or partner

$59(17)$

Median No of years of contact with psychiatric service (interquartile

range)

No of previous psychiatric admissions:

\begin{tabular}{lc}
\hline 0 & $86(25)$ \\
\hline 1 & $85(24)$ \\
\hline 15 & $95(27)$ \\
\hline Unknown & $79(23)$ \\
\hline Main diagnosis*: & $5(1)$ \\
\hline Organic brain syndrome & $5(1)$ \\
\hline Schizophrenia & $84(25)$ \\
\hline Schizoaffective disorder & $20(6)$ \\
\hline Psychotic episode & $77(22)$ \\
\hline Bipolar affective disorder-manic episode & $36(10)$ \\
\hline Bipolar affective disorder-depression episode & $8(2)$ \\
\hline Depression & $71(20)$ \\
\hline Post-traumatic stress disorder & $5(1)$ \\
\hline Personality disorder & $25(7)$ \\
\hline Other & $19(5)$ \\
\hline Alcohol or drug dependent & $43(12)$ \\
\hline Prominent recent history of cannabis use & $79(23)$ \\
\hline Recent history of alcohol misuse & $85(24)$ \\
\hline Recent other substance misuse & $49(14)$ \\
\hline Legal status: & \\
\hline Informal & $197(56)$ \\
\hline Civil detention for assessment (section 2) $\dagger$ & $64(18)$ \\
\hline Civil detention for treatment (section 3) $\dagger$ & $53(15)$ \\
\hline Emergency detention of inpatient $(s e c t i o n ~ 5(2)) \dagger$ & $32(9)$ \\
\hline Emergency detention of outpatient $(s e c t i o n ~ 4) \dagger$ & $3(1)$ \\
\hline Criminal court order & $1(0.3)$ \\
\hline
\end{tabular}

*International classification of diseases, 10 th revision.

†Mental Health Act 1983. (international classification of diseases, 10th revision), and treatment plan. We rated the global assessment of functioning using the Diagnostic and Statistical Manual of MentalDisorders(fourth edition). The clinical researcher (GSO) clarified whether the main decision on treatment was stabilisation with drugs, or admission to a place of safety or for assessment. If the treatment decision was stabilisation with drugs then the assessment of mental capacity centred on the decision to take the prescribed drug or not. If the treatment decision was admission to a place of safety or for assessment then the assessment of mental capacity was to decide whether to come into hospital or not.

\section{Assessments by clinical researcher}

Judgments on mental capacity were based on a clinical assessment (review of notes and clinical interview) and the administration of the MacArthur competence assessment tool for treatment. ${ }^{67}$ This instrument is a semistructured interview that provides disclosures of relevant information to patients about their illness, treatment options, and the risks and benefits of those options. The assessor evaluated the capacity for four abilities relating to the disclosures: understanding, appreciation, reasoning, and expressing a choice. These abilities map onto those regarded as relevant by the Mental Capacity Act 2005.

We modified the MacArthur competence assessment tool for treatment for this study. When the principal decision on treatment concerned stabilisation with drugs, patients were given a disclosure about "no drugs" as the alternative to the "recommended" drugs, rather than other options for drugs. When the principal decision on treatment concerned admission to hospital for safety or assessment, patients were given disclosures about the options of being an inpatient or not. Each disclosure comprised information about the option and the risks and benefits. These changes did not alter the structure of the MacArthur competence assessment tool for treatment. Previous studies have shown excellent inter-rater reliability $(\kappa>0.8)$ for the MacArthur competence assessment tool for treatment used in this way. ${ }^{58}$

\section{Assessments by psychiatric trainees}

Ten psychiatric trainees took part in the study. They were given information on the assessment of mental capacity and received a one hour training session. They were asked to give an opinion on mental capacity to make the key treatment decision on the basis of the patient's presentation at the first interview. This ensured that the treatment decision assessed by the psychiatric trainee was the same as the clinical researcher's and that the assessment was as close to admission as possible.

\section{Statistical analysis}

Analyses were done using Stata $9 \cdot 2$. We estimated the prevalence of mental incapacity in different groups, with $95 \%$ confidence intervals. We compared the 
Table 2 Estimates of prevalence of mental incapacity by diagnosis* and legal status

\begin{tabular}{lcc} 
Sample & No & Prevalence (\%) of incapacity $(95 \% \mathrm{Cl})$ \\
All patients & 338 & $60(55$ to 65$)$ \\
\hline Psychotic illness & 175 & $75(68$ to 81$)$ \\
\hline Schizophrenia & 80 & $81(71$ to 89$)$ \\
\hline Bipolar affective disorder-mania & 36 & $97(86$ to 100$)$ \\
\hline Bipolar affective disorder-depression & 8 & $25(3$ to 65$)$ \\
\hline Depression & 67 & $31(20$ to 44$)$ \\
\hline Personality disorder & 24 & $4(0$ to 21$)$ \\
\hline Informal admission & 188 & $39(32$ to 46$)$ \\
\hline Detained & 150 & $86(79$ to 91$)$
\end{tabular}

*International classification of diseases, 10 th revision.

opinions of the psychiatric trainees with those arising from the research interview, the interview being regarded as the ideal method. We calculated sensitivities and specificities accordingly. Assuming these to be the same for patients seen only by the psychiatric trainees we calculated the expected numbers of patients the clinical researcher would have judged to lack mental capacity in the group seen by the psychiatric trainees. This allowed an estimation of capacity in close to the full sample.

\section{RESULTS}

Figure 1 shows the flow of participants through the study. Overall, 350 people were admitted from the community. Two hundred (57\%) were assessed using the MacArthur competence assessment tool for treatment and $325(93 \%)$ were assessed by psychiatric trainees, of whom 138 (39\%) were assessed only by psychiatric trainees; 12 admissions (3\%) were missed by the clinical researcher and psychiatric trainees.

Table 1 shows the personal, clinical, and legal characteristics of the people admitted. The clinical interviews were held 0 to 8 days after admission (median 2 days, interquartile range 1-3).

Table 2 shows the prevalence of incapacity to make decisions, by diagnosis and legal status. Using sensitivities and specificities of the psychiatric trainees as a test for the research interviewer's ideal in each diagnostic group, and assuming these to be the same for patients seen only by the psychiatric trainees, the prevalence of mental incapacity in the total group $(n=338,97 \%$ of total number of admissions) was estimated to be $60 \%$ (95\% confidence interval 55\% to $65 \%$ ). Most of the detained patients lacked mental capacity.

\section{DISCUSSION}

Lack of mental capacity to make decisions on treatment is common $(60 \%)$ but cannot be presumed in people admitted to psychiatric hospital. It is higher than in patients admitted to a general hospital in the same geographical area. ${ }^{9}$ The prevalence of mental incapacity varied according to diagnosis, with high rates for people with mania and schizophrenia but lower rates for people with depression and personality disorder.

Previous studies used convenience samples or did not include an overall judgment of mental capacity for clinically significant decisions. ${ }^{5}$ Many studies have low participation rates, potentially creating a non-representative sample. Our study overcame these problems, since we achieved a participation rate of $57 \%$ for the full interview, but gained information on nearly all participants, using the psychiatric trainees' assessments. One limitation of the study is that the reported frequencies were from people admitted to psychiatric wards in an urban hospital and may not generalise to non-urban psychiatric settings.

Two fifths of patients admitted voluntarily to psychiatric wards lacked mental capacity. In England and Wales this group is already covered by the Mental Capacity Act 2005, and once the 2007 amendments are fully implemented any "deprivation of liberty" will have to comply with the requirements. The prevalence of mental incapacity in those detained under the Mental Health Act 1983 (not capacity based) is high $(86 \%)$ but not invariable. For inpatients who lack mental capacity the Mental Capacity Act 2005 now provides an alternative statutory framework to the Mental Health Act 1983 for the provision of psychiatric care in England and Wales. Thus to facilitate informed choice between the two acts and in light of the high prevalence of mental incapacity to make decisions in psychiatric inpatients, including those voluntarily admitted, assessment of mental capacity and best interests should become a core part of inpatient psychiatric assessment. Navigating the two legal frameworks, based on different principles, however, is likely to prove complicated.

We thank the psychiatric trainees, the patients, and the ward staff. $\mathrm{MH}$ and ASD are supported by the South London Maudsley NHS Foundation Trust/ Institute of Psychiatry, King's College London, National Institute for Health Research Specialist Biomedical Research Centre.

Contributors: GSO designed the protocol, obtained and analysed the data, coordinated the study and wrote the report. GR, ASD, GS, and PH designed the protocol and edited the report. MH was principal investigator, obtained funding, designed the protocol, analysed data and wrote the report. He is the guarantor

Funding: The Wellcome Trust funded the study and had no role in the design, collection, analysis, or interpretation of data.

Competing interests: GR chaired the expert committee on the review of the Mental Health Act 1983. GR, GS, and ASD have given views to parliamentary committees over reform of the Mental Health Act 1983 when mental capacity has been a central issue. These authors have differing views about mental capacity as a basis for mental health law. GR and GS are in favour of capacity based mental health law. ASD is against. $\mathrm{MH}$ has acted as an expert witness on cases where mental capacity has been under dispute.

\section{WHAT IS ALREADY KNOWN ON THIS TOPIC}

The relation between mental capacity and involuntary psychiatric treatment is ethically controversial but little studied

Mental capacity can be reliably measured

\section{WHAT THIS STUDY ADDS}

Most patients detained under the Mental Health Act 1983 lack mental capacity to make decisions on treatment

A significant proportion of people informally admitted lack mental capacity to make decisions on treatment 
Ethical approval: This study was approved by the joint South London and Maudsley and the Institute of Psychiatry NHS research ethics committee. Provenance and peer review: Not commissioned; externally peer reviewed.

1 Re MB (an adult: medical treatment) [38 BMLR 175] 1997 CA.

Schloerndorff v New York Hospital 1914105 NE 92.

3 Richardson G. Balancing autonomy and risk: a failure of nerve in England and Wales? Int J Law Psychiatry 2007;30:71-80.

4 Cairns R, Maddock C, David AS, Hayward P, Richardson G, Szmukler G, et al. Prevalence and predictors of mental incapacity in psychiatric in patients. Br J Psychiatry 2005;187:379-85.

5 Okai D, Owen G, McGuire H, Singh S, Churchill R, Hotopf M. Mental capacity in psychiatric patients: systematic review. BrJ Psychiatry 2007;191:291-7.
6

Grisso T, Applebaum PS, Hill-Fotouhi C. The MacCAT-T: A clinical tool to assess patients' capacities to make treatment decisions. Psychiatr Serv 1997;48:1415-9.

7 Applebaum PS. Assessment of patient's competence to consent to treatment. N Engl J Med 2007;357:1834-40.

8 Cairns R, Maddock C, David AS, Hayward P, Richardson G, Szmukler G, et al. Reliability of mental capacity assessments in psychiatric inpatients. Br J Psychiatry 2005;187:372-8.

9 Raymont V, Bingley W, Buchanan A, David AS, Hayward P, Wessely S, et al. Prevalence of mental incapacity in medical in-patients and associated risk factors: cross-sectional study. Lancet 2004;364:1421-7.

Accepted: 28 April 2008 